\title{
Odds Ratio, Hazard Ratio and Relative Risk
}

\author{
Janez Stare ${ }^{1}$ \\ Delphine Maucort-Boulch ${ }^{2}$
}

\begin{abstract}
Odds ratio (OR) is a statistic commonly encountered in professional or scientific medical literature. Most readers perceive it as relative risk (RR), although most of them do not know why that would be true. But since such perception is mostly correct, there is nothing (or almost nothing) wrong with that. It is nevertheless useful to be reminded now and then what is the relation between the relative risk and the odds ratio, and when by equating the two statistics we are sometimes forcing OR to be something it is not. Another statistic, which is often also perceived as a relative risk, is the hazard ratio (HR). We encounter it, for example, when we fit the Cox model to survival data. Under proportional hazards it is probably "natural" to think in the following way: if the probability of death in one group is at every time point $k$-times as high as the probability of death in another group, then the relative risk must be $k$, regardless of where in time we are. This could be hardly further from the truth and in this paper we try to dispense with this blunder.
\end{abstract}

\section{Introduction}

\subsection{Relative risk}

In medical studies, probability of seeing a certain event in some group is usually called risk, while epidemiologists might prefer the term incidence (Savitz, 1992). For comparison of risks between groups, the ratio of risks, or the relative risk, is a statistic of choice. Formally, if $\pi_{1}$ is the probability of the event in group 1 , and $\pi_{2}$ is the probability of the event in group 2, then the relative risk is

$$
R R=\frac{\pi_{1}}{\pi_{2}}
$$

The reason of preferring relative risk over the difference of risks

$$
R D=\pi_{1}-\pi_{2}
$$

lies in the fact that the population risks of most diseases are rather small and so differences less dramatic (Walter, 2000). For example, if the probability of some cancer in one group is 0.001 , and in the other 0.009 , the difference is 0.008 (same as between 0.419 and 0.411 ), but the relative risk is 9 !

\footnotetext{
1 Department of Biostatistics and Medical Informatics, University of Ljubljana, Slovenia; janez.stare at mf.uni-lj.si

${ }^{2}$ Service de Biostatistique, Hospices Civils de Lyon, Lyon, France; delphine.maucort-boulch at chu-
} lyon.fr 
Table 1: Probability of death among men and women on the Titanic.

\begin{tabular}{|l|r|r|r|}
\hline Sex & Died & Survived & \multicolumn{1}{c|}{ Risk } \\
\hline men & 1364 & 367 & $1364 / 1731=0.79$ \\
\hline women & 126 & 344 & $126 / 470=0.27$ \\
\hline
\end{tabular}

Table 1 provides an example where the event, unfortunately, was not rare. The relative risk of death of men compared to women is

$$
R R=\frac{0.79}{0.27}=2.93 .
$$

\subsection{Odds ratio}

The other statistics, commonly encountered in medical literature, is the odds ratio (Bland and Altman, 2000). Odds are the ratio of the probability of an event occurring in a group, divided by the probability of that event not occurring

$$
\text { odds }=\frac{\pi}{1-\pi} .
$$

For example, if probability of death in a group is 0.75 , the odds are equal to 3 , since the probability of death is three times higher than the probability of surviving. Table 2 gives the odds among men and women on the Titanic.

Table 2: Odds for death among men and women on the Titanic, $\pi$ denotes the probability of death.

\begin{tabular}{|l|r|r|r|}
\hline & Death & Survival & \\
\hline Sex & $\pi$ & $1-\pi$ & Odds \\
\hline men & 0.79 & 0.21 & 3.76 \\
\hline women & 0.27 & 0.73 & 0.37 \\
\hline
\end{tabular}

If risk was the same in both groups, the odds would be equal. A comparison of odds, the odds ratio, might then make sense.

$$
O R=\frac{\frac{\pi_{1}}{1-\pi_{1}}}{\frac{\pi_{2}}{1-\pi_{2}}}
$$

Odds ratio for the Titanic example is

$$
O R=\frac{3.76}{0.37}=10.16 .
$$

This is very different from the relative risk calculated on the same data and may come as a surprise to some readers who are accustomed of thinking of odds ratio as of relative risk (Greenland, 1987). 
Since we already have relative risk, why would we want to calculate the odds ratio? The answer is not obvious and it is best explained via an example (Nurminen, 1995).

Case-control studies are quite common in medical studies. In these we select a sample of patients and a sample of controls, and study occurrence of some factor, hopefully predictive, in the two groups. The reason for collecting data in such a way is that it takes a long time and big sample sizes to do a follow up study, that is a study in which two groups, with and without a factor, are followed long enough for a disease to appear in numbers large enough to do statistical tests with acceptable power.

Table 3 shows fictional data on prostate cancer and baldness. We see that of the 129 cases, 72 were bald, and 55 were not, while among the 139 controls 82 were bald. Let us remind ourselves that in order to calculate the relative risk between the two groups we would need probabilities of cancer occurring, so probability to have cancer for bald and not bald people. It may seem natural to estimate these probabilities as $\frac{72}{154}$ and $\frac{55}{112}$, and so $R R$ as

Table 3: Prostate cancer and baldness

\begin{tabular}{|l|r|r|r|}
\hline & Case & Control & total \\
\hline bald & 72 & 82 & 154 \\
\hline not bald & 55 & 57 & 112 \\
\hline total & 129 & 139 & 268 \\
\hline
\end{tabular}

$$
R R=\frac{\frac{72}{154}}{\frac{55}{112}}=0.95
$$

but is this correct?

It is very important to understand that this is not correct. Since we randomly chose cases and controls, we can estimate probabilities of observing baldness (or not) among them; but NOT the probabilities of observing cancer among the bald (and not bald) people.

This means that in a study like this we CANNOT calculate the relative risk.

\section{Relative risk and odds ratio ( $R R$ in $O R$ )}

The literature dealing with the relation between relative risk and odds ratio is quite extensive (some examples are (Davies et al., 1998; Deeks, 1998; Newman, 2001; Nurminen, 1995; Pearce, 1993; Savitz, 1992; Zhang and Yu, 1998)). We still hope that the derivation below will be useful.

Table 4 gives a $2 \times 2$ table in general notation.

Using this notation we have

$$
R R=\frac{\frac{n_{11}}{n_{11}+n_{12}}}{\frac{n_{21}}{n_{21}+n_{22}}}=\frac{n_{11}}{n_{21}} \cdot \frac{n_{21}+n_{22}}{n_{11}+n_{12}}
$$

and

$$
O R=\frac{\frac{\pi_{1}}{1-\pi_{1}}}{\frac{\pi_{2}}{1-\pi_{2}}}=\frac{\frac{n_{11} /\left(n_{11}+n_{12}\right)}{n_{12} /\left(n_{11}+n_{12}\right)}}{\frac{n_{21} /\left(n_{21}+n_{22}\right)}{n_{22} /\left(n_{21}+n_{22}\right)}}=\frac{n_{11} n_{22}}{n_{12} n_{21}}
$$


Table 4: A $2 \times 2$ table in general notation.

\begin{tabular}{l|c|c|c} 
& \multicolumn{2}{|c|}{ Outcome } & \\
\hline Factor & Death (Case) & Survival (Control) & Total \\
\hline yes & $n_{11}$ & $n_{12}$ & $n_{11}+n_{12}$ \\
no & $n_{21}$ & $n_{22}$ & $n_{21}+n_{22}$ \\
\hline total & $n_{11}+n_{21}$ & $n_{12}+n_{22}$ & $n$
\end{tabular}

Let us now multiply one column, say cases, by $k$.

Then we have

$$
R R=\frac{k n_{11}}{k n_{21}} \cdot \frac{k n_{21}+n_{22}}{k n_{11}+n_{12}}=\frac{n_{11}}{n_{21}} \cdot \frac{k n_{21}+n_{22}}{k n_{11}+n_{12}}
$$

and

$$
O R=\frac{k n_{11} n_{22}}{n_{12} k n_{21}}=\frac{n_{11} n_{22}}{n_{12} n_{21}} .
$$

We see that the 'relative risk' is now different, but the odds ratio does not change if we change the ratio of cases versus controls.

Until now we have learned the following:

1. we can calculate relative risk IF we can estimate probabilities of an outcome in EACH group.

2. we can't do that in case control studies.

3. we can calculate the odds ratio even if we don't know the probabilities in the groups.

\section{It would then be nice, if odds ratio was close to relative risk.}

Let us now look at the relation between the relative risk and the odds ratio (Zhang and Yu, 1998).

$$
O R=\frac{\frac{\pi_{1}}{1-\pi_{1}}}{\frac{\pi_{2}}{1-\pi_{2}}}=\frac{\pi_{1}}{\pi_{2}} \cdot \frac{1-\pi_{2}}{1-\pi_{1}}=R R \cdot \frac{1-\pi_{2}}{1-\pi_{1}}
$$

From this we see that OR is always further away from 1 than RR. But, more importantly, we see that the odds ratio is close to the relative risk if probabilities of the outcome are small (Davies et al., 1998). And it is this fact that enables us, most of the time, to approximate the relative risk with the odds ratio. Table 5 below illustrates the relationship between RR and OR for some probabilities of the outcome.

\section{Relation between RR and HR}

If one searches the Internet for the relation between the hazard ratio and the relative risk, one will one will predominantly find statements that tell us that these two statistics are more or less equal (Nurminen, 1995). For example, the Glossary at the British Medical Journal site http://clinicalevidence.bmj.com/ceweb/resources/glossary.jsp says 
Table 5: Examples of RR and OR for different probabilities.

\begin{tabular}{|r|r|r|r|}
\hline$\pi_{1}$ & $\pi_{2}$ & RR & OR \\
\hline .4 & .1 & 4 & 6 \\
.2 & .3 & .67 & .58 \\
.04 & .01 & 4 & 4.125 \\
.02 & .03 & .67 & .66 \\
\hline
\end{tabular}

\section{Hazard ratio (HR)}

Broadly equivalent to relative risk (RR); useful when the risk is not constant with respect to time. It uses information collected at different times. The term is typically used in the context of survival over time. If the HR is 0.5 then the relative risk of dying in one group is half the risk of dying in the other group.

The same site has the following definition for Relative Risk

\section{Relative risk (RR)}

The number of times more likely $(R R>1)$ or less likely $(R R<1)$ an event is to happen in one group compared with another. It is the ratio of the absolute risk (AR) for each group. It is analogous to the odds ratio (OR) when events are rare.

Relative risk is calculated as the absolute risk (AR) in the intervention group divided by the AR in the control group.

It would seem that the claim above about HR and RR is generally accepted as correct, although we couldn't find any derivation supporting it. Some of the confusion might be caused by esteemed authors who, in trying to avoid a somewhat unfortunate name proportional hazards model, call such models relative risk models (Kalbfleisch and Prentice, 2002). It is of course obvious that by risk they are referring to the conditional probability of dying in a small interval, so $r(t)=P(t \leq T<t+\Delta t \mid T \geq t)$, but the ratio of such risks is not what people usually understand under the term relative risk, since relative risk is about absolute and not conditional probabilities..

So most of the confusion, or wrong perception, probably comes from this 'natural' line of thought: if hazard ratio is $k$ at all times, then the relative risk must be $k$ at all times. And this is of course wrong.

Relative risk (RR) is a ratio of two probabilities: probability of an event in one group divided by the probability of the same event in the other group. When studying survival, we have to explicitly state in which time interval we are calculating this probability. So, for a given time $t$, the relative risk is

$$
R R(t)=\frac{P\left(T \leq t \mid X=x_{1}\right)}{P\left(T \leq t \mid X=x_{2}\right)}
$$

where $x_{1}$ and $x_{2}$ are values of the covariate $X$ defining the two groups (male, female for example). 
Hazard ratio is a ratio of two hazard functions

$$
H R(t)=\frac{\lambda_{1}\left(t, x_{1}\right)}{\lambda_{2}\left(t, x_{2}\right)}
$$

and we remind the reader that the hazard function is defined as

$$
\lambda(t, x)=\lim _{\Delta t \rightarrow 0^{+}} \frac{P(t \leq T<t+\Delta t \mid T \geq t, X=x)}{\Delta t}
$$

and that hazard is connected to the survival function via the following formula

$$
S(t, x)=e^{-\int_{0}^{t} \lambda(u, x) d u} .
$$

Since

$$
S(t, x)=P(T>t \mid X=x)=1-P(T \leq t \mid X=x)
$$

we can write

$$
R R(t)=\frac{1-S\left(t, x_{1}\right)}{1-S\left(t, x_{2}\right)}=\frac{1-e^{-\int_{0}^{t} \lambda\left(u, x_{1}\right) d u}}{1-e^{-\int_{0}^{t} \lambda\left(u, x_{2}\right) d u}} .
$$

It is difficult to argue that equations (3.1) and (3.2) are similar, but let's try. Sometimes, like in the comparison between the Kaplan-Meier and the Nelson-Aalen estimate of the survival function, the following argument is brought into play

$$
e^{-x} \approx 1-x
$$

This comes from the Taylor series expansion of the function $e^{-x}$ around the value 0

$$
e^{-x}=1-x+\frac{x^{2}}{2}-\cdots
$$

Obviously, approximation (3.3) makes sense only for very small values of $x$. Note that our values of $x$ are $\int_{0}^{t} \lambda\left(u, x_{1}\right) d u$ and $\int_{0}^{t} \lambda\left(u, x_{2}\right) d u$, which are cumulative hazards, increasing without limits when $t$ increases. Such an approximation will never hold, except for early times in a survival study. Applying it (wrongly!) to formula (3.2) in case of proportional hazards, so when $\lambda\left(u, x_{1}\right)=k \lambda\left(u, x_{2}\right)$, would make formulas (3.1) and (3.2) equal.

\section{Illustration}

For easier understanding in Table 6 we give detailed calculations for two groups for the first two times in a possible series of discrete event times and with proportional hazards.

So we have

$$
R R\left(t_{2}\right)=\frac{k\left(p_{1}+p_{2}-k p_{1} p_{2}\right)}{p_{1}+p_{2}-p_{1} p_{2}}
$$

which is NOT equal to $k$, but can be close for small probabilities and small $k$. As time passes, RR is further and further away from HR.

Another example is illustrated in Figure 1 and Table 7. Data were simulated from two exponential distributions with $H R=3$ and with 500 cases in each group. We see that only at the first point, close to $t=0$, the estimate is around 3. Later it quickly diminishes and is already halved at $t=1$. 
Table 6: Calculation of relative risk for at two discrete time points. Hazards are proportional and equal to $k$.

\begin{tabular}{|l|c|c|}
\hline & $t_{1}$ & $t_{2}$ \\
\hline probability of event in group 1 & $p_{1}$ & $p_{2}$ \\
\hline probability of event in group 2 & $k p_{1}$ & $k p_{2}$ \\
\hline probability of survival in group 1 & $1-p_{1}$ & $\left(1-p_{1}\right)\left(1-p_{2}\right)$ \\
\hline probability of survival in group 2 & $1-k p_{1}$ & $\left(1-k p_{1}\right)\left(1-k p_{2}\right)$ \\
\hline probability of event up to $t$ in group 1 & $p_{1}$ & $1-\left(1-p_{1}\right)\left(1-p_{2}\right)$ \\
\hline probability of event up to $t$ in group 2 & $k p_{1}$ & $1-\left(1-k p_{1}\right)\left(1-k p_{2}\right)$ \\
\hline RR up to given time & $\frac{k p_{1}}{p_{1}}=k$ & $\frac{1-\left(1-k p_{1}\right)\left(1-k p_{2}\right)}{1-\left(1-p_{1}\right)\left(1-p_{2}\right)}$ \\
\hline
\end{tabular}

Table 7: Calculation of RR at three different time points for the situation illustrated in Figure 1

\begin{tabular}{|r|r|}
\hline time & RR \\
\hline 0.1 & 3.15 \\
0.5 & 2.12 \\
1.0 & 1.51 \\
\hline
\end{tabular}

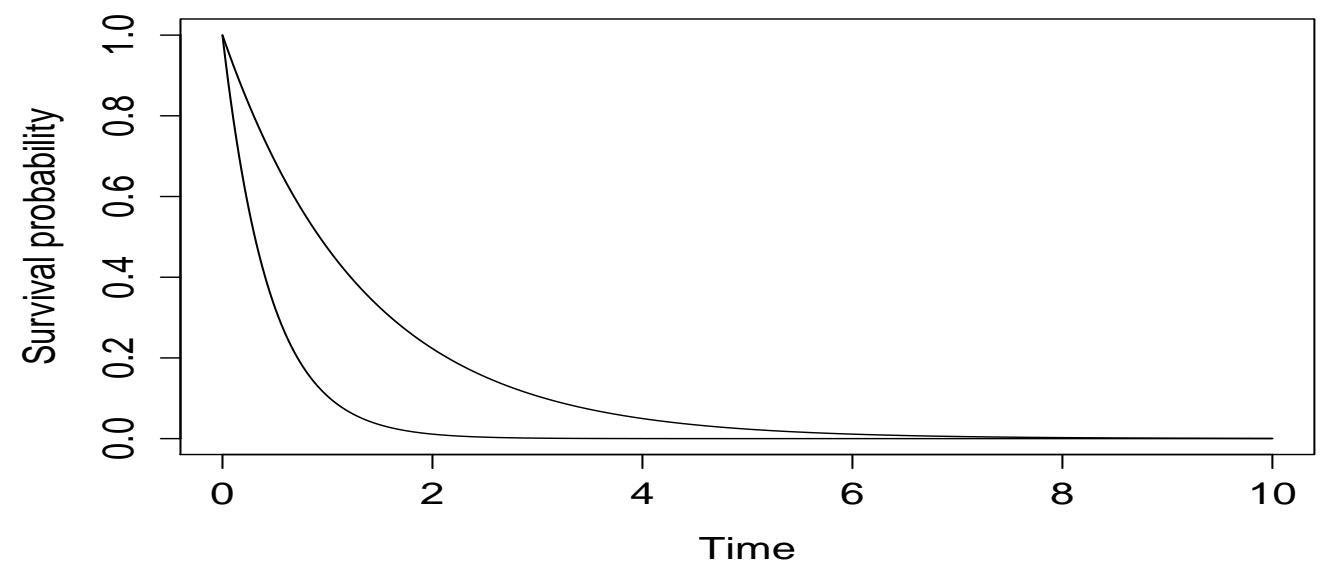

Figure 1: Two exponential curves with $H R=3$. 


\section{Discussion}

In our experience, equating odds ratios with relative risk has become too common, and results, even when probabilities of events are not small, are always interpreted as relative risks (Deeks, 1998; Greenland, 1987; Nurminen, 1995). Having odds ratios as a result of logistic regression fits of course adds to this. We believe that, in case the assumption of a rare event cannot be supported, an effort should be made to estimate relative risk correctly (if possible), or to at least give some estimates, using formula (2.1), for different values of $\pi_{1}$ and $\pi_{2}$.

It is of course possible that many of the claims about the similarity between HR and $\mathrm{RR}$ are made with small intervals in mind. If so, then this should be made very clear when such a statement is made (still, the question why that would be of interest, would remain). The above example from the British Medical Journal site certainly isn't clear about this.

Of course, simply stating that one has small intervals in mind is still not enough. One has to explicitly say that he/she has conditional probabilities in mind as the definition

$$
R R(t)=\frac{P\left(t<T \leq t+\Delta t \mid X=x_{1}\right)}{P\left(t<T \leq t+\Delta t \mid X=x_{2}\right)}
$$

is still NOT the hazard ratio, as it is not a ratio of conditional probabilities.

Maybe the easiest way to understand that a hazard ratio cannot be equal to the relative risk for any time $t$ is to realize that eventually everybody dies, so the relative risk will approach 1 with time, even though the hazard ratio is constant.

\section{References}

[1] Beaudoin, G. (2014): Meeting the information needs of news media to increase citizens' understanding of statistical findings. Paper presented at Work Session on the Communication of Statistics. UNECE.

[2] Bland, J.M. and Altman, D.G. (2000): The odds ratio. British Medical Journal, 320, 1468.

[3] Davies, H.T., Crombie, I.K., and Tavakoli, M. (1998): When can odds ratios mislead? British Medical Journal, 316, 989-991.

[4] Deeks, J. (1998): When can odds ratios mislead? British Medical Journal, 317, $1155-1156$.

[5] Greenland, S. (1987): Interpretation and choice of effect measures in epidemiologic analyses. American Journal of Epidemiology, 125, 761-768.

[6] Kalbfleisch, J.D. and Prentice, R.L. (2002): The Statistical Analysis of Failure Time Data. John Wiley \& Sons.

[7] Newman, S.C. (2001): Biostatistical Methods in Epidemiology. John Wiley \& Sons. 
[8] Nurminen, M. (1995): To use or not to use the odds ratio in epidemiologic studies? European Journal of Epidemiology, 11, 365-371.

[9] Pearce, N. (1993): What does the odds ratio estimate in a case-control study? International Journal of Epidemiology, 22, 1189-1192.

[10] Savitz, D.A. (1992): Measurements, estimates, and inferences in reporting epidemiologic study results. American Journal of Epidemiology, 135, 223-224.

[11] Walter, S.D. (2000): Choice of effect measure for epidemiological data. Journal of Clinical Epidemiology, 53, 931-939.

[12] Zhang, J. and Yu, K.F. (1998): What's the relative risk? A method of correcting the odds ratio in cohort studies of common outcomes. The Journal of the American Medical Association, 280, 1690-1691. 